\title{
Is Higgs inflation ruled out?
}

\author{
Jessica L. Cook, ${ }^{1}$ Lawrence M. Krauss, ${ }^{1,2, *}$ Andrew J. Long, ${ }^{1, \dagger}$ and Subir Sabharwal ${ }^{1}$ \\ ${ }^{1}$ Department of Physics and School of Earth and Space Exploration, \\ Arizona State University, Tempe, Arizona 85827-1404, USA \\ ${ }^{2}$ Research School of Astronomy and Astrophysics, Mt. Stromlo Observatory, \\ Australian National University, Canberra, Australia 2611
}

(Received 20 March 2014; published 22 May 2014)

\begin{abstract}
We consider the status of Higgs inflation in light of the recently announced detection of B modes in the polarization of the cosmic microwave background radiation by the BICEP2 Collaboration. In order for the primordial B-mode signal to be observable by BICEP2, the energy scale of inflation must be high: $V_{\text {inf }} \approx 2 \times 10^{16} \mathrm{GeV}$. Higgs inflation generally predicts a small amplitude of tensor perturbations, and therefore it is natural to ask if Higgs inflation might accommodate this new measurement. We find that the answer is essentially no, unless one considers either extreme fine-tuning or possibly adding new beyond the Standard Model fields, which remove some of the more attractive features of the original idea. We also explore the possible importance of a factor that has not previously been explicitly incorporated, namely the gauge dependence of the effective potential used in calculating inflationary observables (e.g., $n_{S}$ and $r$ ), to see if this might provide additional wiggle room. Such gauge effects are comparable to the effects of Higgs mass uncertainties and other observables already considered in the analysis, and therefore they are relevant for constraining models. However, they are therefore too small to remove the apparent incompatibility between the BICEP2 observation and the predictions of Higgs inflation.
\end{abstract}

DOI: 10.1103/PhysRevD.89.103525

PACS numbers: 98.80.-k

\section{INTRODUCTION}

The theory of inflation [1-3] successfully addressed the 20th century's greatest puzzles of theoretical cosmology. Over the past 20 years, increasingly precise measurements of the temperature fluctuations of the cosmic microwave background $(\mathrm{CMB})$ radiation also confirmed the nearly scale invariant power spectrum of scalar perturbations, a relatively generic inflationary prediction. These many successes, however, underscored the inability to probe perhaps the most robust and unambiguous prediction of inflation, the generation of a background of gravity waves associated with what are likely enormous energy densities concomitant with inflation (e.g., Ref. [4]).

Recently, the BICEP2 Collaboration reported evidence of $\mathrm{B}$ modes in the polarization pattern of the CMB [5]. The B modes result from primordial gravity wave induced distortions at the surface of last scattering. If one assumes that these gravity waves are of an inflationary origin, then the BICEP2 measurement corresponds to an energy scale of inflation,

$$
V_{\mathrm{inf}}^{1 / 4} \approx(2 \pm 0.2) \times 10^{16} \mathrm{GeV},
$$

for a reported tensor-to-scalar ratio of $r \approx 0.2_{-0.05}^{+0.07}$ (using the Planck Collaboration's measurement of the amplitude of the scalar power spectrum [6]). Such a high scale of

\footnotetext{
krauss@asu.edu

† andrewjlong@asu.edu
}

inflation rules out many compelling models. For the purposes of this paper, we will assume that the observation $r \approx 0.2$ is valid,, and we will assess the impact of this measurement on a particular model of inflation, known as Higgs inflation (HI).

Higgs inflation postulates that the Standard Model (SM) Higgs field and the inflaton are one and the same [7]. (See also Ref. [8] for a recent review.) This powerful assumption allows $\mathrm{HI}$ to be, in principle, much more predictive than many other models of inflation, as by measuring the masses of the Higgs boson and the top quark at the electroweak scale $(100 \mathrm{GeV})$, one might predict observables at much larger energy scales associated with inflation $\left(V_{\text {inf }}^{1 / 4} \lesssim 10^{16} \mathrm{GeV}\right)$.

In practice, however, this enhanced predictive power is elusive because of a strong sensitivity to quantum effects, unknown physics, and other technical subtleties in the model. Specifically, one connects observables at the electroweak and inflationary scales using the renormalization group (RG) flow of the SM couplings [9-14]. It is reasonable, however, to expect that there is new physics at intermediate scales, and even if the SM is extended only minimally to include a dark matter candidate [15] or neutrino masses [16-19], this new physics can qualitatively affect the connection between electroweak and inflationary observables. Moreover, perturbative unitarity arguments

\footnotetext{
${ }^{1}$ Note that the BICEP2 measurement is in tension with the upper bound, $r<0.11$ at $95 \%$ C.L., obtained previously by the Planck Collaboration [6].
} 
require new physics just above the scale of inflation [20,21], and the unknown coefficients of dimension six operators can significantly limit the predictive power of $\mathrm{HI}$ [22] as well. The HI calculation also runs into various technical subtleties that arise from the requisite nonminimal gravitational coupling (see below) and quantization in a curved spacetime [23-25]. Finally, it is worth noting that $\mathrm{HI}$ is also at tension with the measured Higgs boson and top quark masses, and an $O(2 \sigma)$ heavier Higgs or lighter top is required to avoid vacuum stability problems [26].

Also, as we shall discuss later in detail, there is one additional source of ambiguity in calculations of $\mathrm{HI}$ that had not been fully explored. Because the quantum corrections are significant when connecting the low energy and high energy observables, one should not work with the classical (tree-level) scalar potential, as is done in many models of inflation; instead, one must calculate the quantum effective potential. It is well known that in gauge theory the effective potential explicitly depends upon the choice of gauge in which the calculation is performed $[27,28]$, and care must be taken to extract gauge-invariant observables from it [29-32] (see also [33,34]). This fact can perhaps be understood most directly by recalling that the effective action is the generating functional for one-particle irreducible Green's functions, which themselves are gauge dependent [28]. In practice, one often neglects this subtlety, fixes the gauge at the start of the calculation, and calculates observables with the effective potential as if it were a classical potential. In the context of finite temperature phase transitions, it is known that when calculated naively in this way, the predictions for observables depend on the choice of gauge used [34-40]. Because of the extreme tension between $\mathrm{HI}$ models and the data, we assess here the degree to which this gauge uncertainty might affect the observables in Higgs inflation. We find that the gauge ambiguity introduces uncertainties that are comparable to the variation of the physical parameters (i.e., the Higgs mass). As a result, this ambiguity alone cannot resuscitate moribund models.

\section{GRAVITY WAVES FROM HIGGS INFLATION}

The Standard Model Higgs potential, $V(h)=\lambda h^{4} / 4$ with $\lambda=O(0.1)$, is too steeply sloped for successful inflation. The measurement of the Higgs boson mass fixes $\lambda \approx 0.13$, whereas $\lambda \ll 1$ is required to produce the observed amplitude of density perturbations. In the HI model, slow roll is achieved by introducing a nonminimal gravitational coupling for the Higgs field, $\mathcal{L}=-\xi \Phi^{\dagger} \Phi R$, where $\Phi$ is the Higgs doublet and $R$ the Ricci scalar. One can remove the nonminimal coupling term from the Lagrangian by performing a conformal transformation, $g_{\mu \nu}(x)=\Omega^{-2}(x) \hat{g}_{\mu \nu}(x)$, where

$$
\Omega^{2}=1+2 \xi \Phi^{\dagger} \Phi / M_{P}^{2}
$$

is the conformal factor and $M_{P}$ is the reduced Planck mass. By doing so, one passes from the Jordan to the Einstein frame. The scalar potential in the new frame becomes

$$
V(h)=\frac{\lambda h^{4}}{4\left(1+\frac{\xi h^{2}}{M_{P}^{2}}\right)^{2}},
$$

where we have written $\Phi^{\dagger} \Phi=h^{2} / 2$. At large field values, $h \gg M_{P} / \sqrt{\xi}$, the potential asymptotes to a constant

$$
V_{0} \approx \lambda M_{P}^{4} / 4 \xi^{2} .
$$

This is the appropriate regime for slow roll inflation.

To evince the tension between Higgs inflation and large tensor perturbations, we can first neglect quantum corrections to $V(h)$ (e.g., the running of $\lambda$ ) as the energy scale of HI, given by Eq. (4), is insensitive to the quantum corrections, whereas the slope is more sensitive.

Since $\lambda$ is fixed by the measured Higgs mass, the scalar potential in Eq. (3) has only one free parameter: $\xi$. It is well known that to achieve sufficient e-foldings of inflation and the correct amplitude for the scalar power spectrum, one needs the nonminimal coupling to be much larger than unity. Specifically, one requires (see, e.g., Ref. [8])

$$
\xi \approx 47000 \sqrt{\lambda},
$$

which is $\xi \approx 17000$ for $\lambda \approx 0.13$. The energy scale of inflation is then predicted to be

$$
V_{0} \approx\left(0.79 \times 10^{16} \mathrm{GeV}\right)^{4},
$$

leading to a tensor-to-scalar ratio, assuming the scalar density perturbations are fixed by $\mathrm{CMB}$ observations, $r \approx 0.0036$. This is naively incompatible with the much larger BICEP2 measurement [see Eq. (1)]. Decreasing $\xi$ in $\mathrm{HI}$ in an attempt to match the newly measured value of $V_{\mathrm{inf}}$ is not workable either, as setting $\xi \approx 2000$ then produces too little power in scalar density perturbations.

Fundamentally, then, the problem in obtaining a large value of $r$ in Higgs inflationary models is that the HI potential asymptotes to a constant at large field values where inflation occurs. This flat potential then results in relatively large density perturbations, which, in order to then match observations, constrain the magnitude of the potential, resulting in a small tensor contribution.

The question then becomes whether variations in this canonical HI-due to quantum effects, for example-will allow the SM Higgs boson to be the inflaton field while also accommodating the large value of $r$.

\section{SAVING HIGGS INFLATION?}

Since it is the nonminimal coupling $\xi$ that flattens out the potential at high scales, one might consider whether there 
are other ways to flatten the potential and thus avoid the requirement for large $\xi$ values.

One possibility proposed in this regard [13] involves fine-tuning the Higgs and top masses such that the Higgs self-coupling runs to a very small value at the scale of inflation, $\lambda \sim 10^{-4}$. This allows for the relatively small $\xi \sim 90$ and produces $r \gtrsim 0.15$. In this region of parameter space, the inflationary observables become strongly dependent on the values of the Higgs and top quark masses. Thus, one can take the measured values of $n_{s}$ and $r$ and use them to derive precise predictions for $m_{h}, m_{t}$, and $\xi$. This procedure favors $m_{t} \approx 171 \mathrm{GeV}$, which is in tension with the current measurement of $173.34 \pm 0.76 \mathrm{GeV}$ [41]. Even in this "tuned" regime, however, it is impossible to entirely eliminate the need for the nonminimal coupling while staying within the Standard Model.

Since the completion of our paper, there have been two new papers further exploring this tuned limit and matching the BICEP2 data [42,43], with even smaller nonminimal couplings $\xi<10$, but with the same caveat of requiring a top mass $3 \sigma$ below its currently measured value.

If one goes outside of the Standard Model, then new physics [44] can affect the running of the Higgs selfcoupling or the anomalous dimension, $\gamma$. For example, one may hope that $\lambda$ or $\gamma$ acquires a significant running at high scales so as to give a workable solution consistent with both the measured scalar and tensor power spectra (see, e.g., Ref. [45]).

As a result, it appears that canonical HI with a nonminimal gravitational coupling as the only new physical input appears extremely difficult to reconcile with the new observation of a large tensor contribution from inflation. It would appear to be necessary to add new physics to eliminate the dependence on nonminimal coupling entirely and to give the Higgs effective potential a shape compatible with observations. Such extensions of HI tend to defeat the original purpose of the idea, namely its predictivity, and most such modifications that have been proposed [46-48] tend to retain the now undesirable feature of small $r$ in any case.

There are two options that might allow large $r$ consistent with BICEP. One possibility involves tuning the Higgs potential to form a second local minimum at large scales, i.e., a false vacuum similar to old inflation [49]. To avoid the problems of old inflation, a time dependent tunneling rate is introduced. While most mechanisms to achieve this, however, produce a small value of $r$ [50], large $r$ can be accommodated by adding a new scalar with a nonminimal coupling to gravity, such that the Higgs field sees a time dependent Planck mass [51]. A second possibility uses a noncanonical Galileon-type kinetic term for the Higgs field. This model yields an $r \simeq 0.14$ [52-54].

These tuned limits, variants, and extensions of the original HI model leave the door slightly open for the possibility of connecting the Higgs with the inflation field.
However, without additional scalars or modification of the Higgs potential via some other mechanism beyond the Standard Model, the original scenario-i.e., Higgs inflation with only a nonminimal coupling to gravity-does not appear to be compatible with the BICEP result.

Before we nail the coffin shut on Higgs inflation, however, there is one possible additional source of uncertainty that merits further investigation. As we describe below, when one goes beyond tree level, there are gauge ambiguities involved in the calculation of effective potentials that need to be considered when deriving constraints on parameters.

\section{GAUGE DEPENDENCE AMBIGUITIES}

When working with a gauge theory, such as the Standard Model electroweak sector, calculations typically involve spurious gauge dependence that is canceled when physical observables are calculated. For example, in a spontaneously broken Yang-Mills theory, one may work in the renormalizable class of gauges $\left(R_{\xi}\right)$ upon augmenting the Lagrangian with a gauge fixing term $\mathcal{L}_{g f}=-G^{a} G^{a} / 2$, where $G^{a}=\left(1 / \sqrt{\xi_{\mathrm{gf}}}\right)\left(\partial_{\mu} A^{a \mu}-\xi \stackrel{\leftrightarrow}{F}_{i}^{a} g \chi_{i}\right), \chi_{i}$ are the wouldbe Goldstone boson fields and $\stackrel{\leftrightarrow}{F}_{i}^{a}=T_{i j}^{a} v_{j}$, with $T_{i j}^{a}$ the symmetry generators and $v_{j}$ the symmetry-breaking vacuum expectation value. (See, e.g., Ref. [33]). A corresponding Fadeev-Popov ghost term is also added. Physical or "on-shell" quantities, such as cross sections and decay rates, may be calculated perturbatively, and any dependence on the gauge fixing parameter $\xi_{\mathrm{gf}}$ cancels order by order. Unphysical or "off-shell" quantities, such as propagators or one-particle irreducible Green's functions, may harmlessly retain the spurious gauge dependence.

The Coleman-Weinberg effective action $\Gamma_{\text {eff }}$ and effective potential $V_{\text {eff }}$ [55] have become standard tools in the study of vacuum structure, phase transitions, and inflation. The effective action is the generating functional of oneparticle irreducible Green's functions; therefore, it is important to recognize that both $\Gamma_{\text {eff }}$ and $V_{\text {eff }}$ are off-shell quantities, which will carry spurious gauge dependence [28]. When applying the effective potential to a problem, special care must be taken to extract gauge-invariant information. In particular, the Nielsen identities express the gauge invariance of the effective potential at its stationary points, but derivatives of the effective potential are not generally gauge invariant [31]. This suggests that inflationary observables, e.g., $n_{S}, r$, and $d n_{S} / d \ln k$, naively extracted directly from the slow roll parameters will acquire a spurious gauge dependence.

Ideally one would like to determine the "correct" procedure for calculating physical quantities like $n_{S}$ from a given model in such a way that the spurious gauge dependence is canceled. There have been significant efforts made in this direction $[23,24]$, but a full gauge-invariant 
formalism has not yet been developed. Here we will take a different approach that is more aligned with recent work on the gauge dependence of phase transition calculations $[34,38,39]$. Specifically, we perform the "naive" HI calculation numerically using the $R_{\xi}$ gauge effective potential and the RG improvement to assess the sensitivity of the inflationary observables to the spurious gauge dependence.

The issues of gauge dependence in the HI calculation were addressed not long after the model was first proposed [56]. Working in the unitary gauge, the authors calculate the RG-improved effective potential for the radial excitation of the Higgs field, and they argue that their calculation is gauge invariant. In particular, the authors contrast their approach with that of Ref. [9], where a qualitatively different relationship between $m_{H}$ and $n_{s}$ was found. In Ref. [9] the RG-improved effective potential depends explicitly on the (non-gauge-invariant) Higgs field anomalous dimension, which follows from solving the CallanSymanzik equation (see Ref. [57]). In Ref. [56] this factor is not included, and consequently their calculation does not inherit its gauge dependence. It is not clear why this factor should be dropped, and more recent work continues to include it (see, e.g., Refs. [13], [15], and [58]). As a result, the issue appears to remain unresolved. We include it here [see Eq. (A6)] and examine the consequences.

We begin by reviewing the familiar Higgs inflation calculation. After moving from the Jordan to the Einstein frame, as described in Sec. II, the resulting action contains a noncanonical kinetic term for the Higgs field. One cannot, in general, find a field redefinition that makes the kinetic term globally canonical [21,59]. At this point, it is customary to move to the unitary gauge where the Higgs doublet is written as $\Phi(x)=e^{2 i \pi^{a}(x) \tau^{a}}(0, h(x) / \sqrt{2})^{T}$. The kinetic term for the radial Higgs excitation can then be normalized by the field redefinition $\chi(h)$, where

$$
d \chi / d h=\sqrt{\frac{1}{\Omega^{2}}+\frac{3}{2} \frac{M_{P}^{2}\left(d \Omega^{2} / d h\right)^{2}}{\Omega^{2}}}
$$

and $\Omega^{2}=1+\xi h^{2} / M_{P}^{2}$.

Having canonically normalized both the gravity and the Higgs kinetic terms, the derivation of the effective potential proceeds along the standard lines. We calculate the RGimproved, one-loop effective potential, as described in the Appendix. Note that we are quantizing the theory and doing all of our calculations in the Einstein frame, as opposed to the Jordan frame, which conforms to conventions in the literature and is consistent with the arguments of Ref. [24]. After performing the RG improvement, the parameter $\lambda$ that appears in Eq. (3) should be understood at the running coupling evaluated at the scale of inflation. Generally, $\lambda<0.1$ and its value depends upon the physical Higgs boson and top quark masses at the input scale. For the bestfit observed values, $M_{H} \approx 125 \mathrm{GeV}$ and $M_{t} \approx 173 \mathrm{GeV}$, the coupling runs negative at $h \approx 10^{10}-10^{12} \mathrm{GeV}$; this is the well-known vacuum stability problem of the Standard Model [26]. Successful HI requires an $O(2 \sigma)$ deviation from central values toward either a larger Higgs boson mass or a smaller top quark mass.

Gauge dependence enters the calculation at two places: explicitly in the one-loop correction to the effective potential and implicitly through the Higgs anomalous dimension upon performing the RG improvement.

To calculate the slow roll parameters, e.g.,

$$
\epsilon=\left.\frac{M_{P}^{2}}{2}\left(V^{\prime} / V\right)^{2}\right|_{h_{\mathrm{cmb}}},
$$

the derivatives are taken with respect to $\chi$, i.e., $V^{\prime}(h(\chi))=(\partial V / \partial h)(d \chi / d h)^{-1}$. The potential and its derivatives are evaluated at the field value, $h_{\mathrm{cmb}}$, for which the number of e-foldings, given by

$$
\mathcal{N}=\int_{h_{\mathrm{end}}}^{h_{\mathrm{cmb}}} d h \frac{V(h)}{V^{\prime}(h) M_{P}^{2}},
$$

is $\mathcal{N}=60$. Inflation terminates at $h=h_{\text {end }}$, where $\left(M_{P}^{2} / 2\right)\left(V^{\prime} / V\right)^{2}=1$.

In Fig. 1 we show the energy scale of inflation,

$$
V_{\mathrm{inf}}=V\left(h_{\mathrm{cmb}}\right)
$$

as the Higgs boson and top quark masses are varied, and the nonminimal coupling, $\xi \approx$ few $\times 10^{3}$, is determined to match the observed amplitude of scalar perturbations. This demonstrates that the scale of inflation is insensitive to $M_{H}$, varying only at the $O\left(10^{-4}\right)$ level. It always remains significantly below $2 \times 10^{16} \mathrm{GeV}$, which indicates the incompatibility with the BICEP2 measurement. (The corresponding tensor-to-scalar ratio is $r \approx 0.003$.)

To illustrate gauge dependence, we show in Fig. 2 how $V_{\text {inf }}$ varies with $\xi_{\text {gf }}$. We find that $V_{\text {inf }}$ also changes at a level comparable to its sensitivity to $M_{H}$ or $M_{t}$ as the gauge parameter deviates from the Landau gauge $\left(\xi_{\mathrm{gf}}=0\right)$. It is

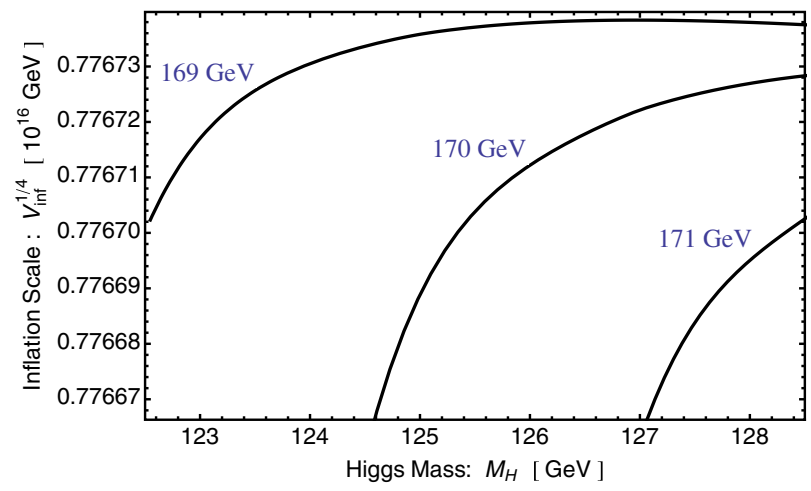

FIG. 1 (color online). The predicted energy scale of inflation, $V_{\text {inf }}^{1 / 4}$, over a range of Higgs boson masses $\left(M_{H}\right)$, for three values of the top quark mass $\left(M_{t}\right)$ and in the Landau gauge, $\xi_{\mathrm{gf}}=0$. 


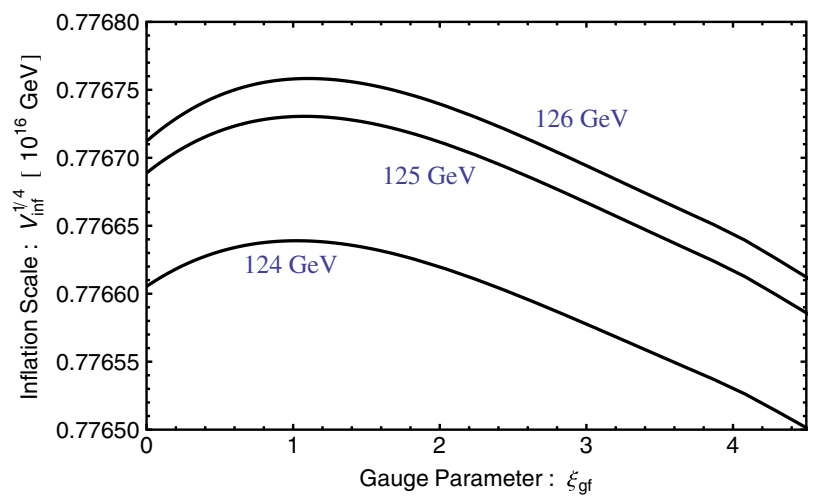

FIG. 2 (color online). The energy scale of inflation, $V_{\text {inf }}$, as the gauge parameter $\xi_{\mathrm{gf}}$ varies. We fix $M_{t}=170 \mathrm{GeV}$ and show three values of $M_{H}$.

therefore important to consider this ambiguity for model building purposes. Nevertheless, the absolute change in $V_{\text {inf }}$ is far too small to reconcile HI with the BICEP2 measurement. Note that at larger values of $\xi_{\mathrm{gf}}$, the scale of inflation appears to continue to decrease, but in this limit the perturbative validity of the calculation begins to break down. To resolve this issue, the unphysical degrees of freedom, the Goldstone bosons and ghosts, should be decoupled as the unitary gauge is approached.

Our numerical results appear consistent with the Nielsen identities [31,32], which capture the gauge dependence of the effective potential. The relevant identity is

$$
\left[\xi_{\mathrm{gf}} \frac{\partial}{\partial \xi_{\mathrm{gf}}}+C\left(\phi, \xi_{\mathrm{gf}}\right) \frac{\partial}{\partial \phi}\right] V_{\mathrm{eff}}\left(\phi, \xi_{\mathrm{gf}}\right)=0 .
$$

In the slow roll regime, the gradient of the effective potential is small and the gauge dependence is proportionally suppressed. However, it is important to stress that gauge-invariant information can be extracted from the effective potential only at an extremum. Since the inflaton is displaced from an extremum during slow roll, the calculation necessarily acquires some degree of spurious gauge dependence.

A more rigorous gauge-invariant approach requires the full effective action (see, e.g., Refs. [23,24]). It could perhaps take Eq. (11), or the corresponding identity for the effective action, as a starting point. This might be an interesting avenue for future work, either in the context of $\mathrm{HI}$ or for other, potentially more viable models of inflation that are embedded in gauge theories.

\section{CONCLUSION}

The recent detection of $\mathrm{B}$ modes by the BICEP2 Collaboration represents a profound and exciting leap forward in our ability to explore fundamental physics and the early Universe. If the measurement of $r \approx 0.2$ is confirmed, then it is reasonable to expect that, in the not-too-distant future, measurements of the spectrum of primordial tensor perturbations will become possible, thereby allowing for further tests of inflation. If the measured $r$ can unambiguously be shown to be due to inflation, then this also substantiates the quantization of gravity [60].

Thus, future observations will provide significant constraints on particle physics and models of inflation. However, the simple observation of nonzero $r$ already signals the death knell for low-scale models of inflation. This includes the class of models captured by the potential in Eq. (3), and among these, apparently, Higgs inflation. We have shown that $r \approx 0.2$ essentially excludes canonical Higgs inflation in the absence of extreme fine-tuning. The Higgs field may live on as the inflaton, but only with significant nonminimal variants of $\mathrm{HI}$.

In our analysis, we have also drawn attention to the issue of gauge dependence in the Higgs inflation calculation. We find that the energy scale of inflation acquires an artificial dependence on the gauge fixing parameter by virtue of the gauge dependence of the effective potential from which it is extracted. However, we find that this gauge dependence of the scale of inflation is comparable to the dependence on other physical parameter uncertainties, which are themselves small. While this may be important for model building purposes, it does not affect the robustness of the fact that a large $r$ disfavors Higgs inflation.

\section{ACKNOWLEDGMENTS}

This work was supported by ANU and by the U.S. DOE under Grant No. DE-SC0008016. We would like to thank Jayden L. Newstead for help with the code.

\section{APPENDIX: STANDARD MODEL EFFECTIVE POTENTIAL}

The Standard Model effective potential is calculated (i) to the one-loop order, (ii) working in the $\overline{\mathrm{MS}}$ renormalization scheme with renormalization scale $\mu$, and (iii) in the renormalizable class of gauges $\left(R_{\xi}\right)$, as follows:

$$
V_{\text {eff }}(h)=V^{(0)}(h)+V^{(1)}(h) .
$$

The tree-level potential is

$$
V^{(0)}(h)=\frac{\lambda}{4} h^{4},
$$

and we can neglect the $O\left(h^{0}\right)$ and $O\left(h^{2}\right)$ terms for the purposes of studying HI where the field value is large. The one-loop correction is [61] (see also [34] for gauge dependent factors) 


$$
\begin{aligned}
V^{(1)}(h) & -\frac{12}{4} \frac{\tilde{m}_{t}^{4}}{16 \pi^{2}}\left(\ln \frac{\tilde{m}_{t}^{2}}{\mu^{2}}-\frac{3}{2}\right)+\frac{6}{4} \frac{\tilde{m}_{W}^{4}}{16 \pi^{2}}\left(\ln \frac{\tilde{m}_{W}^{2}}{\mu^{2}}-\frac{5}{6}\right) \\
& +\frac{3}{4} \frac{\tilde{m}_{Z}^{4}}{16 \pi^{2}}\left(\ln \frac{\tilde{m}_{Z}^{2}}{\mu^{2}}-\frac{5}{6}\right)+\frac{1}{4} \frac{\tilde{m}_{G}^{4}}{16 \pi^{2}}\left(\ln \frac{\tilde{m}_{G}^{2}}{\mu^{2}}-\frac{3}{2}\right) \\
& +\frac{2}{4} \frac{\tilde{m}_{G^{ \pm}}^{4}}{16 \pi^{2}}\left(\ln \frac{\tilde{m}_{G^{ \pm}}^{2}}{\mu^{2}}-\frac{3}{2}\right)-\frac{2}{4} \frac{\tilde{m}_{c_{W}}^{4}}{16 \pi^{2}}\left(\ln \frac{\tilde{m}_{c_{W}}^{2}}{\mu^{2}}-\frac{3}{2}\right) \\
& -\frac{1}{4} \frac{\tilde{m}_{c_{Z}}^{4}}{16 \pi^{2}}\left(\ln \frac{\tilde{m}_{c_{Z}}^{2}}{\mu^{2}}-\frac{3}{2}\right),
\end{aligned}
$$

where we have neglected the light fermions. We also neglect the contribution from the Higgs mass term. During inflation, the potential is very flat and this contribution is subdominant. The remaining SM fields, the massless photon and gluons, do not enter the effective potential at the one-loop order. The effective masses are

$$
\begin{array}{rlrl}
\text { Top Quark } & \tilde{m}_{t}^{2}=\frac{y_{t}^{2}}{2 \Omega^{2}} h^{2}, \\
\text { W Bosons } & \tilde{m}_{W}^{2}=\frac{g^{2}}{4 \Omega^{2}} h^{2}, \\
\text { Z Bosons } & \tilde{m}_{Z}^{2}=\frac{g^{2}+g^{2}}{4 \Omega^{2}} h^{2}, \\
\text { Higgs Boson } & \tilde{m}_{H}^{2}=\frac{3 \lambda}{\Omega^{4}} h^{2} \frac{1-\xi h^{2} / M_{P}^{2}}{\Omega^{2}+6 \xi^{2} h^{2} / M_{P}^{2}}, \\
\text { Neutral Goldstone } & \tilde{m}_{G}^{2}=\frac{\lambda}{\Omega^{4}} h^{2}+\tilde{m}_{c_{Z}}^{2}, \\
\text { Charged Goldstones } & \tilde{m}_{G^{ \pm}}^{2}=\frac{\lambda}{\Omega^{4}} h^{2}+\tilde{m}_{c_{W}}^{2}, \\
\text { Ghosts } & \tilde{m}_{c_{Z}}^{2}=\xi_{\mathrm{gf}} \tilde{m}_{Z}^{2}, \\
\text { Ghosts } & \tilde{m}_{c_{W}}^{2} & =\xi_{\mathrm{gf}} \tilde{m}_{W}^{2},
\end{array}
$$

where $\Omega^{2}=1+\xi h^{2} / M_{P}^{2}$ was given by Eq. (2). We denote the gauge fixing parameter by $\xi_{\mathrm{gf}}$ to distinguish it from the nonminimal gravitational coupling parameter $\xi$. We implement the RG improvement as per [62-64] (see also the reviews [57,61]). This consists of (i) solving the RG equations (RGEs) to determine the running parameters as functions of the RG flow parameter $t$, (ii) replacing the various coupling constants in $V_{\text {eff }}$ with the corresponding running parameter, and (iii) evaluating the RG flow parameter at the appropriate value $t=t_{*}$ so as to minimize the would-be large logarithms. For the sake of discussion, let us denote the running parameters collectively as $\hat{c}_{i}(t)=\left\{\hat{g}_{3}(t), \hat{g}_{2}(t), \hat{g}_{1}(t), \hat{\lambda}(t), \hat{y}_{t}(t), \hat{\xi}(t)\right\}$, where $g_{2}=g$ and $g_{1}=g^{\prime}$. The RGEs then take the form $\beta_{\hat{c}_{i}} /(1+\gamma)=d \hat{c}_{i} / d t$, with the boundary condition $\hat{c}_{i}(t=0)=c_{i, 0}$. Here $\gamma$ is the anomalous dimension of the Higgs field. We neglect the running of the gauge fixing parameter $\xi_{\mathrm{gf}}$ since it is self-renormalized. This approximation is reasonable since we focus on $\xi_{\mathrm{gf}}<4 \pi$; for larger values of $\xi_{\mathrm{gf}}$, perturbativity becomes an issue. The Higgs field runs according to $-\gamma \hat{h}=d \hat{h} / d t$, where the anomalous dimension $\gamma(t)$ is given as [65]

$$
\begin{aligned}
\gamma= & \frac{1}{(4 \pi)^{2}}\left[-\frac{9}{4}\left(1-\frac{\xi_{\mathrm{gf}}}{3}\right) g_{2}^{2}-\frac{3}{4}\left(1-\frac{\xi_{\mathrm{gf}}}{3}\right) g_{1}^{2}+3 y_{t}^{2}\right] \\
& -\frac{1}{(4 \pi)^{4}}\left[\left(\frac{271}{32}-3 \xi_{\mathrm{gf}}-\frac{3}{8} \xi_{\mathrm{gf}}^{2}\right) g_{2}^{4}-\frac{9}{16} g_{1}^{2} g_{2}^{2}-6 s^{3} \lambda^{2}\right. \\
& \left.-\frac{431}{96} s g_{1}^{4}-\frac{5}{2}\left(\frac{9}{4} g_{2}^{2}+\frac{17}{12} g_{1}^{2}+8 g_{3}^{2}\right) y_{t}^{2}+\frac{27}{4} s y_{t}^{4}\right] .
\end{aligned}
$$

This last equation may be solved immediately, along with the boundary condition $\hat{h}(t=0)=h_{c}$, to obtain

$$
\hat{h}(t)=h_{c} e^{\hat{\Gamma}(t)},
$$

where $\hat{\Gamma}(t)=-\int_{0}^{t} \gamma\left(t^{\prime}\right) /\left(1+\gamma\left(t^{\prime}\right)\right) d t^{\prime}$; we seek to calculate the effective potential as a function of $h_{c}$. The beta functions are independent of $\xi_{\mathrm{gf}}$, but the anomalous dimension is gauge variant since the Higgs field is a gauge-variant operator. Finally, the renormalization scale runs according to $\hat{\mu}=d \hat{\mu} / d t$, which may be solved along with $\hat{\mu}(t=0)=\mu_{0}$ to obtain $\hat{\mu}(t)=\mu_{0} e^{t}$. We solve the one-loop beta functions using the Mathematica code made publicly available by Fedor Bezrukov at http://www.inr.ac .ru/ fedor/SM/. The code implements the matching at the electroweak scale to determine the couplings $c_{i, 0}$ at the scale $\mu_{0}=M_{t}$ in terms of the physical masses and the parameters. The code was extended (i) by generalizing the anomalous dimension to the $R_{\xi}$ gauge, as in Eq. (A5), and (ii) by including the field-dependent factors of

$$
s=\frac{1+\frac{\hat{\xi}(t) \hat{h}(t)^{2}}{M_{P}^{2}}}{1+(1+6 \hat{\xi}(t)) \frac{\hat{\xi}(t) \hat{h}(t)^{2}}{M_{P}^{2}}}
$$

in the two-loop beta functions, as indicated by [13]. The factor of $s$ arises because of the noncanonical Higgs kinetic term, and it appears in the commutator of the Higgs field with its conjugate momentum [9]. Finally, the RGimproved effective potential is evaluated as in Eq. (A1) after making the replacements $\lambda \rightarrow \hat{\lambda}\left(t_{*}\right), g \rightarrow \hat{g}\left(t_{*}\right)$, $h \rightarrow \hat{h}\left(t_{*}\right), \mu \rightarrow \hat{\mu}\left(t_{*}\right)$, and so on. The RG flow parameter $t_{*}$ is chosen to minimize the would-be large logarithm arising from the top quark. This is accomplished by solving

$$
\left.\frac{\hat{y}_{t}(t)^{2} \hat{h}(t)^{2}}{2\left(1+\frac{\hat{\xi}(t) \hat{h}(t)^{2}}{M_{P}^{2}}\right) \hat{\mu}(t)^{2}}\right|_{t=t_{*}}=1,
$$

which must be done numerically. Note that $t_{*}$ is an implicit function of the field variable $h_{c}$. This can be seen by writing 


$$
t_{*}=\frac{1}{2} \ln \left[\frac{\hat{y}_{t}\left(t_{*}\right)^{2} e^{2 \hat{\Gamma}\left(t_{*}\right)} h_{c}^{2}}{2 \mu_{0}^{2}}\right] \approx \frac{1}{2} \ln \left[\frac{y_{0}^{2} h_{c}^{2}}{2 \mu_{0}^{2}}\right] .
$$

Using Eq. (A8), the commutator factor in Eq. (A7) is written as

$$
s=\left[1+12 \frac{\hat{\xi}(t)^{2} \hat{\mu}(t)^{2}}{\hat{y}_{t}^{2} M_{P}^{2}}\right]^{-1},
$$

and the field dependence drops out.
[1] A. A. Starobinsky, Phys. Lett. B 91, 99 (1980).

[2] A. H. Guth, Phys. Rev. D 23, 347 (1981).

[3] A. D. Linde, Phys. Lett. B 108, 389 (1982).

[4] L. M. Krauss and M. J. White, Phys. Rev. Lett. 69, 869 (1992).

[5] BICEP2 Collaboration, arXiv:1403.3985.

[6] P. Ade et al. (Planck Collaboration), arXiv:1303.5082.

[7] F. Bezrukov and M. Shaposhnikov, Phys. Lett. B 659, 703 (2008).

[8] F. Bezrukov, Classical Quantum Gravity 30, 214001 (2013).

[9] A. De Simone, M. P. Hertzberg, and F. Wilczek, Phys. Lett. B 678, 1 (2009).

[10] A. Barvinsky, A. Y. Kamenshchik, and A. Starobinsky, J. Cosmol. Astropart. Phys. 11 (2008) 021.

[11] F. Bezrukov, A. Magnin, M. Shaposhnikov, and S. Sibiryakov, J. High Energy Phys. 01 (2011) 016.

[12] F. Bezrukov, M. Y. Kalmykov, B. A. Kniehl, and M. Shaposhnikov, J. High Energy Phys. 10 (2012) 140.

[13] K. Allison, J. High Energy Phys. 02 (2014) 040.

[14] A. Salvio, Phys. Lett. B 727, 234 (2013).

[15] T. Clark, B. Liu, S. Love, and T. ter Veldhuis, Phys. Rev. D 80, 075019 (2009).

[16] N. Okada, M. U. Rehman, and Q. Shafi, arXiv:0911.5073.

[17] B. He, N. Okada, and Q. Shafi, Phys. Lett. B 695, 219 (2011).

[18] W. Rodejohann and H. Zhang, J. High Energy Phys. 06 (2012) 022.

[19] A. Kobakhidze and A. Spencer-Smith, J. High Energy Phys. 08 (2013) 036.

[20] J. Barbon and J. Espinosa, Phys. Rev. D 79, 081302 (2009).

[21] C. Burgess, H. M. Lee, and M. Trott, J. High Energy Phys. 07 (2010) 007.

[22] C. Burgess, S. P. Patil, and M. Trott, arXiv:1402.1476.

[23] D. P. George, S. Mooij, and M. Postma, J. Cosmol. Astropart. Phys. 11 (2012) 043.

[24] D. P. George, S. Mooij, and M. Postma, J. Cosmol. Astropart. Phys. 02 (2014) 024.

[25] T. Prokopec and J. Weenink, arXiv:1403.3219.

[26] D. Buttazzo, G. Degrassi, P. P. Giardino, G. F. Giudice, F. Sala, A. Salvio, and A. Strumia., J. High Energy Phys. 12 (2013) 089.

[27] S. Weinberg, Phys. Rev. D 7, 2887 (1973).

[28] R. Jackiw, Phys. Rev. D 9, 1686 (1974).

[29] L. Dolan and R. Jackiw, Phys. Rev. D 9, 2904 (1974).

[30] J. Kang, Phys. Rev. D 10, 3455 (1974).

[31] N. K. Nielsen, Nucl. Phys. B101, 173 (1975).

[32] R. Fukuda and T. Kugo, Phys. Rev. D 13, 3469 (1976).
[33] I. J. R. Aitchison and C. M. Fraser, Ann. Phys. (N.Y.) 156, 1 (1984).

[34] H. H. Patel and M. J. Ramsey-Musolf, J. High Energy Phys. 07 (2011) 029.

[35] S. Weinberg, Phys. Rev. D 9, 3357 (1974).

[36] L. Dolan and R. Jackiw, Phys. Rev. D 9, 3320 (1974).

[37] C. W. Bernard, Phys. Rev. D 9, 3312 (1974).

[38] C. Wainwright, S. Profumo, and M. J. Ramsey-Musolf, Phys. Rev. D 84, 023521 (2011).

[39] C. L. Wainwright, S. Profumo, and M. J. Ramsey-Musolf, Phys. Rev. D 86, 083537 (2012).

[40] M. Garny and T. Konstandin, J. High Energy Phys. 07 (2012) 189.

[41] ATLAS Collaboration, CDF Collaboration, CMS Collaboration, and D0 Collaboration, arXiv:1403.4427.

[42] Y. Hamada, H. Kawai, K.-y. Oda, and S. C. Park, arXiv: 1403.5043.

[43] F. Bezrukov and M. Shaposhnikov, arXiv:1403.6078.

[44] V. Branchina and E. Messina, Phys. Rev. Lett. 111, 241801 (2013).

[45] J. Joergensen, F. Sannino, and O. Svendsen, arXiv: 1403.3289.

[46] O. Lebedev and H. M. Lee, Eur. Phys. J. C 71, 1821 (2011).

[47] V. V. Khoze, J. High Energy Phys. 11 (2013) 215.

[48] Y. Hamada, H. Kawai, and K.-y. Oda, Prog. Theor. Exp. Phys. (2014) 023B02.

[49] I. Masina and A. Notari, Phys. Rev. Lett. 108, 191302 (2012).

[50] I. Masina and A. Notari, J. Cosmol. Astropart. Phys. 11 (2012) 031.

[51] I. Masina and A. Notari, Phys. Rev. D 85, 123506 (2012).

[52] K. Kamada, T. Kobayashi, M. Yamaguchi, and J. Yokoyama, Phys. Rev. D 83, 083515 (2011).

[53] K. Kamada, T. Kobayashi, T. Takahashi, M. Yamaguchi, and J. Yokoyama, Phys. Rev. D 86, 023504 (2012).

[54] K. Kamada, T. Kobayashi, T. Kunimitsu, M. Yamaguchi, and J. Yokoyama, Phys. Rev. D 88, 123518 (2013).

[55] S. R. Coleman and E. J. Weinberg, Phys. Rev. D 7, 1888 (1973).

[56] F. Bezrukov and M. Shaposhnikov, J. High Energy Phys. 07 (2009) 089.

[57] C. Ford, D. Jones, P. Stephenson, and M. Einhorn, Nucl. Phys. B395, 17 (1993).

[58] R. N. Lerner and J. McDonald, Phys. Rev. D 83, 123522 (2011).

[59] D. I. Kaiser, Phys. Rev. D 81, 084044 (2010).

[60] L. M. Krauss and F. Wilczek, Phys. Rev. D 89, 047501 (2014). 
[61] M. Sher, Phys. Rep. 179, 273 (1989).

[62] B. M. Kastening, Phys. Lett. B 283, 287 (1992).

[63] M. Bando, T. Kugo, N. Maekawa, and H. Nakano, Phys. Lett. B 301, 83 (1993).
[64] M. Bando, T. Kugo, N. Maekawa, and H. Nakano, Prog. Theor. Phys. 90, 405 (1993).

[65] A. Bednyakov, A. Pikelner, and V. Velizhanin, Nucl. Phys. B875, 552 (2013). 